\title{
Compressed Sensing Reconstruction of Remote Sensing Image Block based on Augmented Lagrangian Method TV
}

\author{
Sheng Cang ${ }^{\mathrm{a}, \mathrm{b}}$ and Achuan Wang ${ }^{\mathrm{a}, *}$ \\ ${ }^{a}$ Northeast Forestry University, Harbin, 150040, China \\ ${ }^{b}$ Heilongjiang International University, Harbin, 150025, China
}

\begin{abstract}
With the development of remote sensing technology and the diversification of sensors, remote sensing image data reveals the trend of "three features" -- high resolution, hyper spectral and multi-temporal. As the increasing demand of remote sensing information, considerable amounts of data will be acquired, transmitted and stored in various remote sensing applications, which, without doubt, sets higher requirements for data processing. To solve the above problems, according to the feature of compressed sensing theory, which original image can be reconstructed by low sampling data, we develop a new method of Remote Sensing Image Block Compressed Sensing Reconstruction Based on Augmented Lagrangian Method TV. It represented remote sensing image sparsely by means of block sampling and joint sparse representation model. Besides, it also combined the total Variation and Augmented Lagrangian method to optimize the solution and implemented the algorithm of the model. Finally, it created a remote sensing image with low distortion. Furthermore, it also increased efficiency in data transmission and reduced data storage. Simulation test results confirm the validity of algorithm proposed in this paper and also suggest that it can achieve better effects of a distinct advantage in PSNR, which is remote sensing image reconstruction, in comparison with other algorithms.
\end{abstract}

Keywords: Nyquist; remote sensing image; compressed sensing; image reconstruction; augmented lagrangian method TV

(Submitted on November 8, 2017; Revised on January 2, 2018; Accepted on February 3, 2018)

(C) 2018 Totem Publisher, Inc. All rights reserved.

\section{Introduction}

Remote sensing technology is a kind of detection technology dating back to 1960s. This technology, based on electromagnetic wave theory, transmits electromagnetic waves with different wavebands to target objects to be detected, by which images could be formed on the basis of the recognition and processing of radiation and reflection information. Various types of transmitters could be used to transmit electromagnetic waves; thus, the remote sensing detection of target objects at a long distance or range could be achieved. These devices vary the feedback, including an infrared ray satellite nephogram, visible light and electromagnetic wave. With the advances in science and technology, the remote sensing transmission devices in the past have been replaced by satellite remote sensing ones. Since satellite features real time, stability, long distance and high power, modern remote sensing technology is gradually dominated by the satellite remote sensing technology, which includes steps such as sending, receiving, transmitting and processing. Among them, the receiver includes synthetic aperture radar (SAR), microwave radiometer (MR), imaging spectrometer (IS), multi-spectral scanner (MSS), TV-camera and camera. The transmission equipment includes radar used for transmission, the processing device includes digital image processor, image inter-pre to scope and additive color viewer $[6,12]$. Remote sensing image features a large amount of data acquisition and high dimension as well as a low speed of data acquisition. However, most of the traditional method of data processing was based on Shannon-Nyquist sampling theorem. It seeks to keep the important information from original signal complete, which requires that the signal sampling frequency must be higher than twice the signal bandwidth. Traditional Nyquist sampling, due to the limit of its sampling rate, leads to problems such as long data acquisition time, low data transmission efficiency and information storage redundancy. But in the actual environment, to acquire information based on Nyquist sampling theorem, there needs a higher sampling rate and in order to complete large numbers of tasks such as the compression,

\footnotetext{
* Corresponding author.

E-mail address: achuanwang@126.com
} 
transmission and storage of considerable original information; the hardware performance should be continuously improved. Nevertheless, this will increase the cost and waste of resources. Therefore, sampling at high rates and then compression coding based on Nyquist sampling technology can consume so many resources that it would be difficult to meet requirements such as processing considerable information with high quality in future remote sensing applications. However, the presence of Compressed Sensing Theory offers a new way to address the problem, which can transmit and store considerable remote sensing image data efficiently.

CS theory, proposed by Cades, Romberg, Tao and Donoho in 2006, is a new information acquisition theory that can finish both signals sampling and compression at the same time, which was a great breakthrough in signal acquisition technology $[3,5,9]$. It indicates that important information from original signal can be kept complete by a few random projections of sparse or compressible signals. Then, the target problem can be optimized by adopting the reconstruction algorithm; in this way, original signal might be reconstructed with high probability from low sampling measurements $[10,11,16,17]$. The difference between CS theory and Nyquist sampling theorem is the signal sparseness. It can acquire data at much lower sampling rate than that stated by Nyquist sampling theorem and can also acquire data as few as possible so as to include large numbers of important information, thus reconstructing original signal accurately or appreciatively, which brings a new idea and method to image processing field. The core idea of the CS theory mainly includes two aspects. The first aspect is its irrelevance, that is, for compressed sensing, the collected signal information can be optimized by the algorithm to achieve its irrelevance, so that dealing with compressed samples can be achieved by continuous time signal, during the process of which redundant information can be discarded directly. In this way, the amount of data required to deal with can be reduced. In addition, in the process of obtaining the sparse signal, it is possible to realize its approximate sparse sampling process by a non-adaptive sampling method. The second aspect is the sparse structure. Since remote sensing information has some structural characteristics, the sparse signal can be achieved by priori information, so that redundant information can be removed. In the process of information reconstruction, only a few digital codes and a small amount of priori information, the information to be collected can be restored. Since the emergence of CS theory, various theories and application researches on CS spring up, including the analysis of the theoretical basis, signal sparse representation, the construction and optimization of the measurement matrix, the emergence of various reconstruction methods and its applications in different fields. Currently, overseas researchers have applied it to the remote sensing, mainly involving fusion of remote sensing images, classification and identification, super-resolution reconstruction and so on. Although the research has achieved certain results, it is still immature. Literature $[1,7,13]$ is based on wavelet transform sparsity reconstruction model, under which remote sensing image reconstruction is achieved by improving the weak segmentation threshold of conjugate gradient tracking algorithm; the reconstruction accuracy and the speed of algorithms are improved. Among most of the existing remote sensing image reconstruction methods, sparse representation is based on fixed basis functions, which has ignored the own sparsity of remote sensing image, thus leading to less effective reconstruction and limiting the further development of CS theory in remote sensing applications.

In this paper, a new method of remote sensing image block compressed sensing and reconstruction based on ALMT is proposed. Based on block sampling and improved joint sparsity representation model, the remote sensing image can be more sparsely expressed by adopting an augmented Lagrangian ALMT method based on ALM and TV method, an efficient solution, which is a remote sensing image reconstruction algorithm with high efficiency and low distortion.

\section{Related Work}

\subsection{Basic Theory of Compressed Sensing}

When signal is compressible or in some sparse transform domains, theory of compressed sensing can collect little precision or approximated reconstructed original signal in measurement from linear projection. For any n-dimensional signals $U \in R^{N}$ , they can represent sparsely $u=\psi \alpha$ in fundamental space $\psi \in R^{N \times N}, \quad \alpha$ is the $x$ of transform coefficient vector in $\psi$ domain. If there is only $K$ non-zero elements in transform coefficient $\alpha$, or just maintain most component of $K$, ( $N>>K$ ) ,the signal x will be sparsely $K$ or approximated sparsely k[2,4,8,14,15].

To be more specific, define a matrix with $M \times N$-dimension $(M<<N)$ random projection. Signal u sampled few of M-dimensional measurement $\mathrm{b}, b=A u$. $\mathrm{b}$ is sampling signal from the original signal u compressed sample meanwhile. It completely maintain the important information of original signal $\mathrm{u}$. Compressed sensing theory is aimed at making use of a few of measurements $b$ after compressed sample to rebuild original signal $u$. It represents constrained optimization problems below. It is shown in Equation (1). 


$$
\min _{u}\|\psi u\|_{p} \quad \text { s.t. } \quad b=A u
$$

In Equation (1), combinational optimization problem is the reconstruction of sparse signal $\mathrm{u}$. The problem is called NPhard and cannot find solution straightly. In equation, $p$ is 1 or $0, \mid * \|_{1}$ is the norm of 1 . It represents the sum of all elements' absolute value in the vector. $\mid * \|_{0}$ is the norm of 0 and represents the number or non-zero elements in the vector.

\subsection{Augmented Lagrangian Method}

Considering a constrained optimization problem, it is shown in Equation (2).

$$
\min _{f} E(f) \quad \text { s.t. } \quad H f=g
$$

In equation, $f \in R^{N}, g \in R^{N}, \quad H \in R^{M \times N}$.

The definition of Augmented Lagrange method objective function is below, it is shown in Equation (3).

$$
\ell_{A}(f, \lambda)=E(f)-\lambda^{T}(H f-g)+\frac{\mu}{2}\|H f-g\|_{2}^{2}
$$

In equation, $\lambda \in R^{M}$ is Augmented Lagrangian method's factor. $\mu \geq 0$ is parameter with a penalty term. The core idea of Augmented Lagrange Method is efficient combination between standard Lagrangian function and penalty function. When penalty parameter is sufficiently high, it will find objective function's optimal solution with the help of the Lagrangian multiplier iteration. In Equation (3) $\ell_{A}(f, \lambda)$, that's equal to the saddle point of to solve the constrained optimization problem. The difference between Augmented Lagrangian function and standard Lagrangian function is that the former adds a penalty term, and containing the liner term of multiplier $\lambda$ is the distinction between Augmented Lagrangian function and penalty function. ALM is a kind of representative optimizes method. It provided a way to solve the optimizing of remote sensing image reconstruction. The method is update $\lambda$ and minimize objective function's constrained optimization problems. Repeat these two steps until it meet the convergence criterion. The detail description is shown in Algorithm1.

Algorithm 1 Augmented Lagrangian method (ALM)

Input: Compressed sensing measurement $\mathrm{b}$ and linear measurement matrix $A, \beta, \theta, \mu, \omega$.Initialization.

Output: Remote sensing image $\mathrm{u}$ is finally reconstructed.

1. Set $k=0$, choose $\mu>0, f_{0}$ and $\lambda_{0}$

2. When the out loop termination condition is not satisfied "do"

3. When the inner loop termination condition is not satisfied "do"

4. Use Equation $f_{k+1} \in \arg \min _{f} \ell_{A}\left(f, \lambda_{k}\right)$ to solve the sub problem $f_{k+1}$

5. Use Equation $\lambda_{k+1}=\lambda_{k}-\mu\left(H f_{k+1}-g\right)$ to solve the sub problem $\lambda_{k+1}$

6. Use Equation $k \leftarrow k+1$ to solve the sub problem $k$

7. The inner loop is end

8. The outer loop is end.

\section{The Method of Remote Sensing Image Reconstruction Base on Block Compressed Sensing}

The tendency of "three features" in remote sensing data development brings a high data rate to remote sensing image. In order to reduce complexity of calculation and increase speed of calculation, this paper uses the thought of block compressed sensing and TV method to realize the reconstructive algorithm of remote sensing image. First, it is based on block sampling, divided original remote sensing image into some non-overlapped image pieces in same size, and obtained improved joint sparse representation model by basic priori knowledge. Random sampling is implemented in each sub-images. Finally, with the use of TV method and ALM reconstructive algorithm, scarce of measurements are obtained to rebuild original remote sensing image from all piece of image in compressed sampling. 


\subsection{Improved Joint Sparse Representation Model}

Say concretely, $\mid \psi_{\mathrm{L} 2 \mathrm{D}} u \|_{1}$ is corresponding to the local smoothness future knowledge, keeping the local continuity of image so that noise can be effectively suppressed. Built on the assumption that the gradient image of the remote sensing image is Laplace distribution. The sacristy of the local 2D spatial domain is characterized by using the image of the remote sensing image to be spared, which mathematical expression shows below. It is shown in Equation (4).

$$
\left\|\psi_{\mathrm{L} 2 \mathrm{D}} u\right\|_{1}=\|D u\|_{1}=\left\|D_{v} u\right\|_{1}+\left\|D_{h} u\right\|_{1}
$$

Here, D means the gradient operator, $D_{v}, D_{h}$ mean the horizontal and vertical gradient operator respectively. However, $\mid \psi_{\mathrm{N} 3 \mathrm{D}} u \|_{0}$ correspond the future knowledge of nonlocal-means self-similarities. Maintain image non local continuity. Image sharpness and edge can be effectively preserved. Processing at the image block level, to every piece of image $x^{i}, \mathrm{i}=1,2, \ldots \mathrm{n}$, first to search windows to find c similar block $S x^{i}$, The similar image blocks $S x^{i}$ generated by the superposition are transformed into three dimensional groups $Z x^{i}$. The transformation ratio is $T^{3 D}\left(Z_{x^{i}}\right)$.It is got from the three-dimensional array with the Orthogonal 3D. Finally, the coefficients of the three-dimensional groups are combined by the combination of all the analogous image blocks. Characterize the global self-similarity of non-local 3D transform domain, its mathematical is shown in Equation (5).

$$
\left\|\psi_{\mathrm{N} 3 \mathrm{D}} x\right\|_{0}=\left\|\Theta_{x}\right\|_{0}=\sum_{i=1}^{n}\left\|T^{3 D}\left(Z_{x^{i}}\right)\right\|_{0}
$$

Here, $\theta_{x}$ means image block $\mathrm{x}$-- the column vectors of all transform coefficients. A more detailed mathematical description, please consult the reference.

\subsection{Model optimization algorithm of the ALMT}

Substitute the result (3)(4)(5) into (1), on the basis of retaining the important information of the original image and reducing the distortion of images, describe the Inherent sparsity of images to get the objective function on the basic of remote sensing image reconstruction problem with compressive sensing joint sparsity. It is shown in Equation (6).

$$
\min _{u} \tau \cdot\|D u\|_{1}+\lambda \cdot\left\|\Theta_{u}\right\|_{0} \quad \text { s.t. } \quad A u=b
$$

Reconstruct objective Equation (6) is hard to solve the problem directly. Here show implementation details of effective algorithm application of ALM optimization algorithm. First, through introducing auxiliary variables $w, x$ to translated (7) into an equivalent Equation as follow. It is shown in Equation (7).

$$
\underset{w, u, x}{\operatorname{argmin}} \tau \cdot\|w\|_{1}+\lambda \cdot\left\|\Theta_{x}\right\|_{0} \text { s.t. } D u=w, u=x, A u=b
$$

According to Equation (3), by Equation (8) corresponding to the augmented Lagrange function.

$$
\ell_{A}(w, u, x)=\tau\|\mathrm{w}\|_{1}-v^{T}(D u-w)+\frac{\beta}{2}\|D u-w\|_{2}^{2}+\lambda\left\|\Theta_{x}\right\|_{0}-\gamma^{T}(u-x)+\frac{\theta}{2}\|u-x\|_{2}^{2}-\rho^{T}(A u-b)+\frac{\mu}{2}\|A u-b\|_{2}^{2}
$$

Here $\beta, \theta, \quad \mu$ correspond to $\|D u-w\|_{2}^{2},\|u-x\|_{2}^{2},\|A u-b\|_{2}^{2}$ respectively. ALM method's key is to find out $\ell_{A}(w, u, x)$ to achieve the optimum solution of Equation (7). The following two steps achieve the ALM method to solve the constrained optimization problem. It is shown in Equation (9).

$$
\left(w_{k+1}, u_{k+1}, x_{k+1}\right)=\underset{w, u, x}{\operatorname{argmin}} \ell_{A}(w, u, x)
$$




$$
\left\{\begin{array}{c}
v_{k+1}=v_{k}-\beta\left(D u_{k+1}-w_{k+1}\right) \\
\gamma_{k+1}=\gamma_{k}-\theta\left(u_{k+1}-x_{k+1}\right) \\
\rho_{k+1}=\rho_{k}-\mu\left(A u_{k+1}-b\right)
\end{array}\right.
$$

In Equation (10), $k, v, \quad \gamma$, and $\rho$ respectively correspond to constraint term Lagrange multiplier of $D u=w, u=x, A u=b$. According to Equation (10), it should adopt alternating direction method, fix other variables, minimize a variably alternately, and transform into three sub-problem $w, u, x$. Given a constant $u$ and $x$, the optimizations question about $w$ is. It is shown in Equation (11).

$$
\min _{w} \delta_{1}(\mathrm{w})=\min _{w}\left\{\tau\|w\|_{1}-v^{T}(D u-w)-\frac{\beta}{2}\|D u-w\|_{2}^{2}\right\}
$$

According to [16,17], the approximate expression of Equation (11) is. It is shown in Equation (12).

$$
\tilde{w}=\max \left\{\left|D u-\frac{v}{\beta}\right|-\frac{\tau}{\beta}, 0\right\} \cdot \operatorname{sgn}\left(D u-\frac{v}{\beta}\right)
$$

Where, the dot is meant by inner product of the two vectors. Given a constant $w$ and $x$, the optimization problem about $u$ is below. It is shown in Equation (13).

$$
\min _{u} \delta_{2}(u)=\min _{u}\left\{-v^{T}(D u-w)+\frac{\beta}{2}\|D u-w\|_{2}^{2}-\gamma^{T}(u-x)+\frac{\theta}{2}\|u-x\|_{2}^{2}-\rho^{T}(A u-b)+\frac{\mu}{2}\|A u-b\|_{2}^{2}\right\}
$$

Obviously, Equation (14) is a quadratic function, solve it with the method below:

$$
\tilde{\mathrm{u}}=u-\eta d
$$

In the Equation, D as the objective function (14) gradient $\eta$ is the optimal descent step, where I is the unit matrix: $\eta=a b s\left(d^{T} d / d^{T} G d\right), G=\left(\beta D^{T} D+\theta I+\mu A^{T} A\right) \mathrm{d}=D^{T}(\beta D u-v)-\gamma+\theta(u-x)-\beta D^{T} w+A^{T}(\mu(A u-b)-\rho)$. Given $W, u$, then the optimization problem about $X$ is as follows. It is shown in Equation (15).

$$
\min _{x} \delta_{3}(x)=\min _{x}\left\{\lambda\left\|\Theta_{x}\right\|_{0}-\gamma^{T}(u-x)+\frac{\theta}{2}\|u-x\|_{2}^{2}\right\}
$$

On the Equation (15) application of soft threshold, and omitted the independent constant x, and get Equation (16).

$$
\min _{x} \delta_{3}(x)=\min _{x}\left\{\frac{\theta}{2}\|x-r\|_{2}^{2}+\lambda\left\|\Theta_{x}\right\|_{0}\right\}=\min _{x}\left\{\frac{1}{2}\|x-r\|_{2}^{2}+\frac{\lambda}{\theta} \sum_{k=1}^{n}\left\|T^{3 D}\left(Z_{x_{k}}\right)\right\|_{0}\right\}
$$

In this Equation, $r=(u-\gamma / \theta)$. As $\Theta_{x}$ exist, Equation (16) is still hard to solve. For ease of handling. Assume $\mathrm{r}$ is $\mathrm{x}$, which is some type of noise observation value and let $e=x \quad r$. Every factor of e follows variance is $\sigma^{2}$, Independent distribution of zero mean and no need for Gauss process. Based on the above assumptions, because of $e, x, r \in R^{N}, \Theta_{r}$, $\Theta_{r} \in R^{k} \Theta_{r} \in R K$, according to the theorem of large numbers. The existence of the maximum probability of the existence of the following two equations was established. It is shown in Equation (17).

$$
\frac{1}{N}\|x-r\|_{2}^{2}=\sigma^{2}, \frac{1}{K}\left\|\Theta_{x}-\Theta_{r}\right\|_{2}^{2}=\frac{1}{K} \sum_{k=1}^{n}\left\|T^{3 D}\left(Z_{x_{k}}\right)-T^{3 D}\left(Z_{r_{k}}\right)\right\|_{2}^{2}=\sigma^{2}
$$


Combine (16) and (17) to get Equation (18).

$$
\min _{x} \delta_{3}(x)=\min _{x} \frac{1}{2}\left\|\Theta_{x}-\Theta_{r}\right\|_{2}^{2}+\frac{N \lambda}{K \theta}\left\|\Theta_{x}\right\|_{0}
$$

In Equation (18), the approximate expression could be $\tilde{\Theta}_{x}=\operatorname{hard}\left(\Theta_{r}, \sqrt{2 \omega}\right)$, where $\operatorname{hard}(z, a)=\max \{a b s(z-a), 0\} \cdot \operatorname{sgn}(z)$ and $\omega=K \lambda / N \theta$, finally to get the Equation (19) about $\mathrm{X}$, as follow:

$$
\tilde{x}=\Omega_{N 3 D} \tilde{\Theta}_{x}=\Omega_{N 3 D}\left(\operatorname{hard}\left(\Theta_{r}, \sqrt{2 \omega}\right)\right)
$$

\section{Experimental results and analysis}

We utilize four pictures to do the experiment. Figure 1 is the four pictures. The computer we use is Dual core CPU and the Matlab vision is 7.12. Firstly, the image was dissolved into $128 * 128$ sub-image. Secondly, Gaussian random projection matrix was adopted to sample randomly each sub-image.

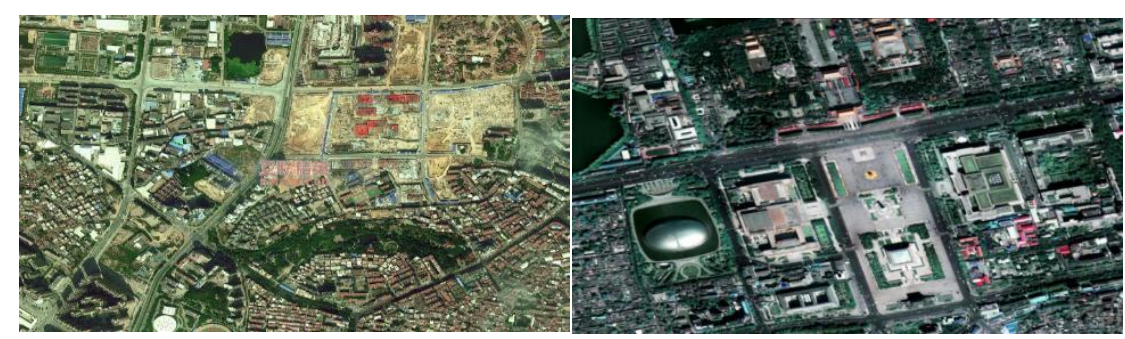

(a) General

(b) Tiananmen
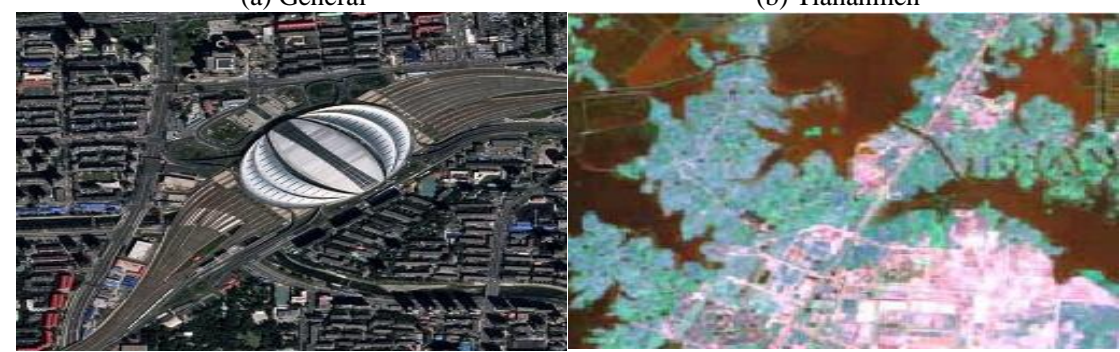

(c)Station

(d)Wuhan

Figure 1. The four pictures of remote sensing image

\begin{tabular}{|c|c|c|c|c|c|}
\hline Figure & $\begin{array}{l}\text { Rate of the above } \\
\text { image }\end{array}$ & TV Method & MH Method & CoS Method & ALMT Method \\
\hline \multirow[t]{4}{*}{ General } & 0.3 & 10.75 & 18.37 & 18.55 & 19.49 \\
\hline & 0.5 & 14.35 & 21.62 & 22.14 & 23.13 \\
\hline & 0.7 & 19.28 & 25.28 & 26.17 & 27.46 \\
\hline & 0.9 & 20.65 & 26.72 & 28.51 & 30.28 \\
\hline \multirow{4}{*}{ Tiananmen } & 0.3 & 18.69 & 20.67 & 22.89 & 25.42 \\
\hline & 0.5 & 20.51 & 22.52 & 25.91 & 27.98 \\
\hline & 0.7 & 26.38 & 27.37 & 30.07 & 31.55 \\
\hline & 0.9 & 28.15 & 29.28 & 31.52 & 33.33 \\
\hline \multirow{4}{*}{ Station } & 0.3 & 17.26 & 18.55 & 19.34 & 20.22 \\
\hline & 0.5 & 19.53 & 20.62 & 21.34 & 23.42 \\
\hline & 0.7 & 20.12 & 21.32 & 22.63 & 24.31 \\
\hline & 0.9 & 29.28 & 30.12 & 32.22 & 33.78 \\
\hline \multirow{4}{*}{ Wuhan } & 0.3 & 18.87 & 19.21 & 20.32 & 20.85 \\
\hline & 0.5 & 19.28 & 20.37 & 21.55 & 24.27 \\
\hline & 0.7 & 20.31 & 21.23 & 22.53 & 26.28 \\
\hline & 0.9 & 24.26 & 25.13 & 26.32 & 30.28 \\
\hline \multicolumn{2}{|c|}{ Average } & 20.48 & 23.02 & 24.44 & 25.82 \\
\hline
\end{tabular}

Table 1. The Result of PSNR 
Finally, restructuring algorithm was used to compress restructure the little measure results from the samples to generate remote image restructuring. Through the experimental analysis the regularization item $r$ is $0.625, \lambda$ being 0.375 . It is shown in Figure 1. The sample rate of the above image is $0.2,0.3,0.5$, and 0.8 of compressed sampling. The calculation method is restructuring method. To prove the effectiveness of the calculation method, Total Variation (TV) method, Multi-Hypothesis (MH) method, and Collaborative sparse $(\mathrm{CoS})$ was adopted to restructure the restoration of the above images. To evaluation the capacity of different calculating methods, the PSNR of each restructuring was measured. The higher the PSNR, the higher the quality of the restructuring image is. PSNR result appears in Table 1.

From the result of the calculation, in different sampling rate, comparing with TV, MH and CoS, the restructuring method adopted in the experiment gained high PSNR. On average, the adopted calculating method is respectively $5.34 \mathrm{~dB}$ and $2.8 \mathrm{~dB}$ higher than TV and MH. Comparing to the CoS, the method proposed increasing 1.38dB. Figure 2, Figure 3 and Figure 4 show the curve of the PSNRs for the four remote sensing images of the sampling rates of $0.3,0.5,0.7$ and 0.9 , respectively.

The curve of the PSNR (GENERAL)

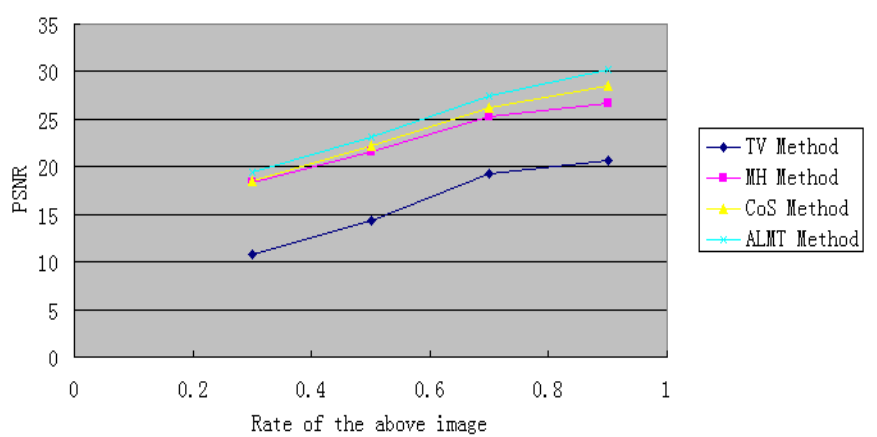

Figure 2. The curve of the PSNR (GENERAL)

As is shown in Figure 2, with the increase of sampling rate, the PSNR of ALMT algorithm is higher than that of TV, MH and Cos. Among them, the PSNT of TV is the lowest, and is much lower than the other three algorithms.

As is shown in Figure 3, with the increase of sampling rate, when the PSNR of ALMT algorithm is 0.3, 0.5, 0.7 and 0.9, the corresponding PSNR of it is higher than those of TV, MH and CoS. According to the analysis of this figure, ALMT reconstruction effect is the best.

The curve of the PSNR(Tiananmen)

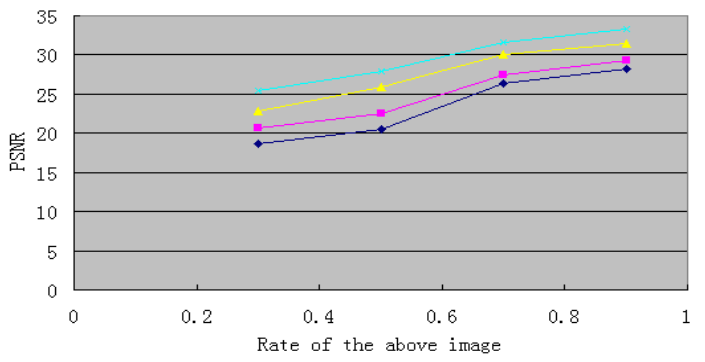

Figure 3. The curve of the PSNR (Tiananmen)

The curve of the PSNR(Station)

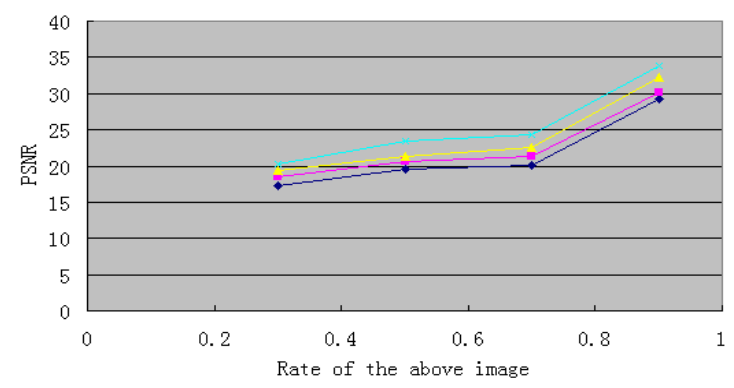

Figure 4. The curve of the PSNR (Station)

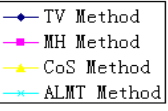

$\rightarrow-$ TV Method

MH Method

CoS Method
- ALMT Method 
As shown in Figure 4, with the increase of sampling rate, the corresponding PSNR of ALMT is greater than the other three algorithms. Since the texture characteristics of the figure are clear, there are no great differences among these algorithms. As shown in Figure 5, with the increase of sampling rate, the PSNR of ALMT is higher than the other three algorithms. Because of the obvious structure, the difference of PSNR among TV, MH and Cos algorithm is not significant, but when sampling rate of the ALMT algorithm in this paper are $0.5,0.7$ and 0.9 . Its effect is obviously better than the other three algorithms.

The curve of the PSNR(Wuhan)

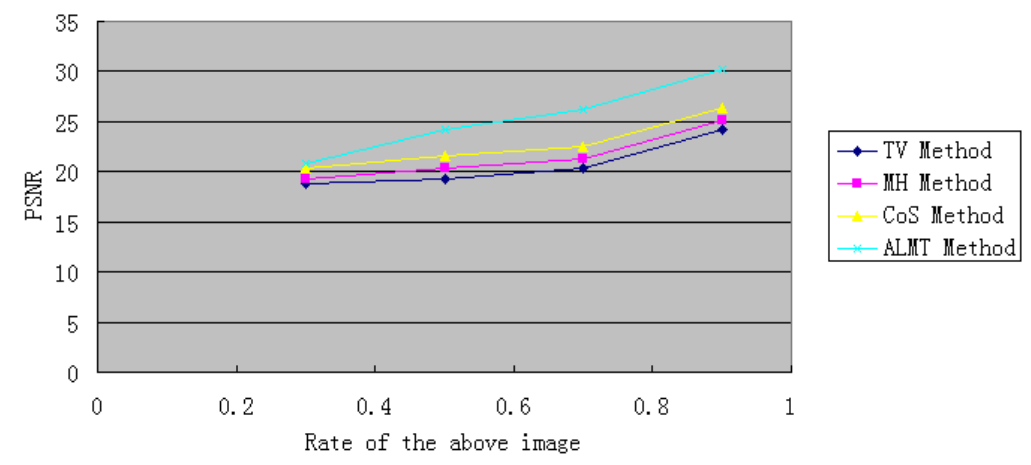

Figure 5. The curve of the PSNR (Wuhan)

However, in a subjective vision, as shown in Figure 6 and Figure 7, they are respectively Beijing South Station remote image and Wuhan remote image under the sampling rate of 0.5 . The experimental restructuring results from different restructuring methods are depicted. It can be seen that TV effect was not as good as that of the MH and CoS. However, the former restructuring image was blurry, whereas the latter was not as thorough. The experimental method, on the other hand, generated clearer images and can to an extent keep complete image details and structure. From the experiment result, the proposed restructuring method is better than $\mathrm{TV}, \mathrm{MH}$, and $\mathrm{CoS}$ being correct and effective. As there still exist the lack of thoroughness of $\mathrm{CoS}$, the experiment method utilizes two regularization parameters to better balance the entire sparse of a remote image, to effectively keep image details and edge improving the quality of the restructuring image.

Block sampling cannot only increase the sampling rate, but also resolute the definition of the images. Cos, on the other hand, processes the entire image compressed cognition sampling. TV processing result replaces ' 0 ' initialization to realize the proposed experimental restructuring method. Iterations are largely minimized to gain a better effect. Hundreds of minimized of $\mathrm{CoS}$ were reduced to dozens of minimized so as to reduce the sense of compression and restructuring time to increase the speed of calculation. Therefore, the proposed method is more advantageous in remote image restructuring effect and speed. Ultimately, the astronomical definition remote image restructuring is generated.
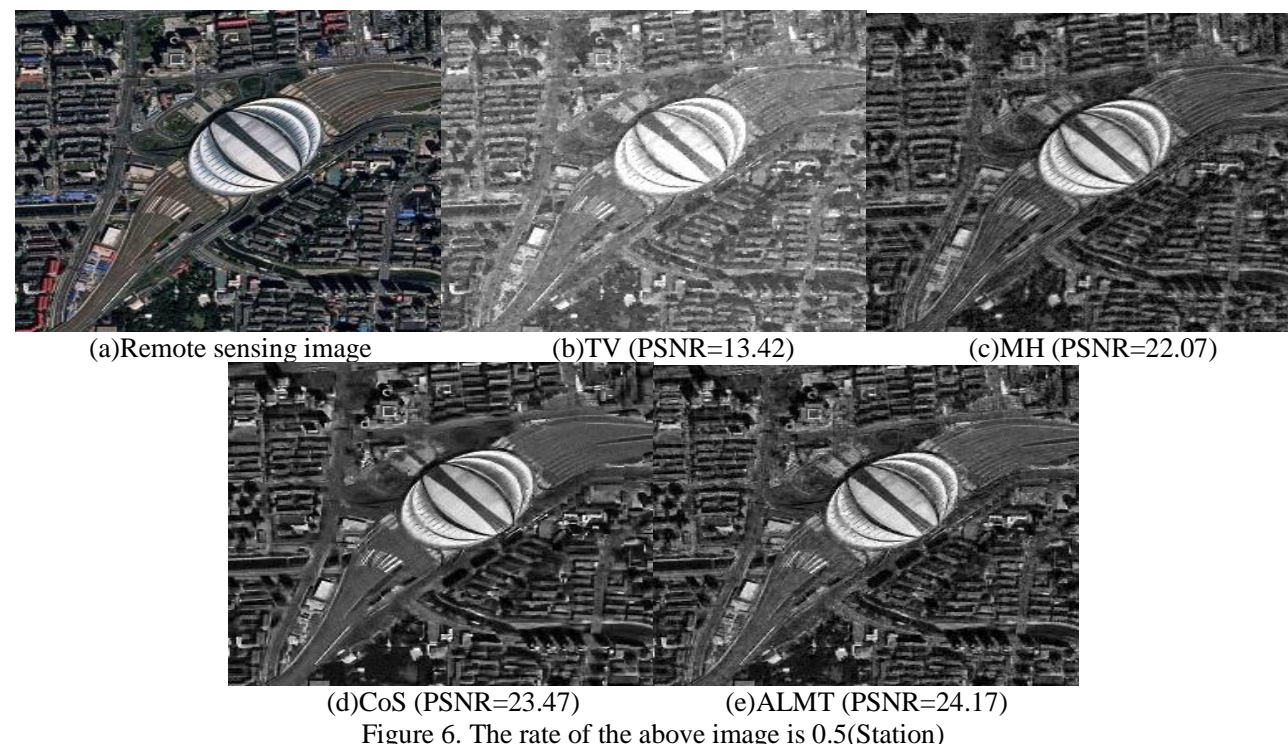

Figure 6. The rate of the above image is 0.5 (Station) 

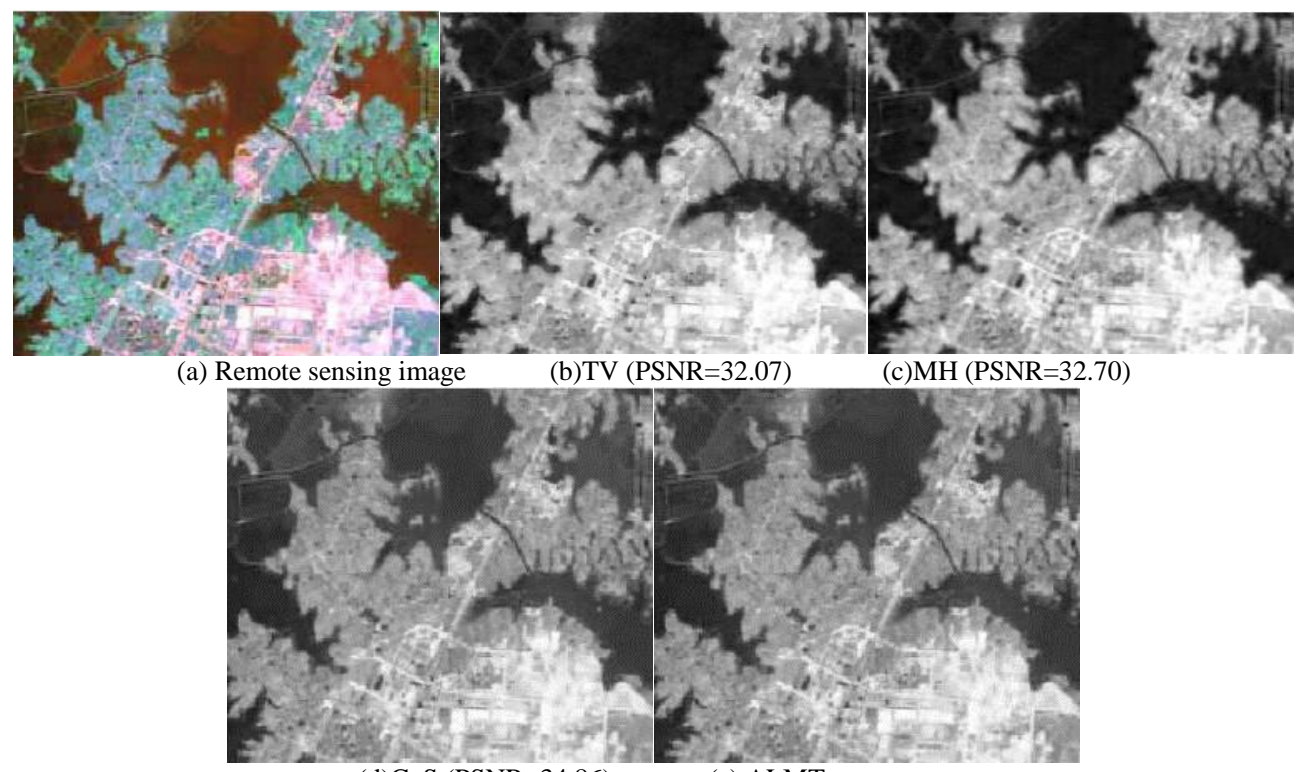

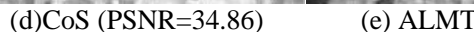

Figure 7. The rate of the above image is 0.5 (Wuhan)

\section{Conclusions}

Concerning the waste of resource of remote image vast data sampling, transferring and storing, a new ALMT method is suggested, block compression cognition remote image restructuring. Through two regularisation parameters to respectively gain the knowledge of remote image, and to balance the sparse of the image, the model of improved joint sparse was gained. Under the block sampling, the sub-images were sampled. Combining TV and ALM, effective solution was carried out for improved joint sparse expression model and calculation realization was conducted. The experiment result indicates that the method suggested enables the remote image to be sparser and improves adaptability. Comparing with CoS, the PSNR in the method has increased $1 \mathrm{~dB}$ and reduced calculating amount as well as data storage capacity realising a high sampling rate and high definition remote image restructuring. The proposed method is more advantageous in remote image restructuring effect and speed providing a new method for transferring and storing data of remote image.

\section{References}

1. V. Afonso, "Fast Image Recovery Using Variable Splitting and Constrained Optimization", IEEE Transactions on Image Processing, vol. 9, no. 19, pp.2345-2356,2010

2. C. Chen, E. Tramel, "Compressed-sensing Recovery of Images and Video Using Multi-hypothesis Predictions", In Proceedings of the 45th Asilomar Conference on Signals, Systems, and Computers, Pacific Grove, USA November, pp.22-25,2015

3. E. J. Candes, J. Romberg, "Robust Uncertainty Principles: Exact Signal Reconstruction From Highly Incomplete Frequency Information”, IEEE Transactions on Information Theory, vol. 2, no. 3, pp. 489-509,2013.

4. J. Cai and Z. Shen, "Split Bregman Methods and Frame Based Image Restoration", Multiscale Modeling and Simulation, vol. 2, no. 8 , pp, 337-36,2015

5. D. Donoho, “Compressed sensing”, IEEE Transactions on Information Theory, vol. 4, no. 3, pp. 1289-1306,2014

6. S. Gu, L. Zhang, W. Zuo and X. Feng, "Weighted Nuclear Norm Minimization with Application to Image Denoising",IEEE Conference on Computer Vision and pattern recognition, vol. 2, no. 6, pp. 2862-2869,2014

7. L. He, L. Carin, "Exploiting Structure in Wavelet-based Bayesian Compressive Sensing", IEEE Transactions on Signal Processing, vol. 3, no. 12,pp. 3488-3497,2015

8. Y. Kim, "Compressed Sensing Using a Gaussian Scale Mixtures Model in Wavelet Domain", In Proceedings of International Conference on Image Processing, Hongkong, China,2014

9. H. Liu, R. Xiong, S. Ma, X. Fan and W. Gao, "Non-local Extension of Total Variation Regularization for Image Restoration", IEEE International Symposium on Circuits and Systems, Chennai, India, February ,pp.27-28,2014

10. H. Liu, R. Xiong, S. Ma, X. Fan and W. Gao, "Gradient Based Image Transmission and Reconstruction Using Non-local Gradient Sparsity Regularization", IEEE International Conference on Multimedia and Expo, Chengdu, China, January,pp.12-14,2014

11. H. Liu, R. Xiong, S. Ma, X. Fan and W. Gao, "Gradient Based Image/Video Soft Cast with Grouped-patch Collaborative Reconstruction", IEEE Visual Communications and Image Processing Conference, Valletta, Malta, December,pp.5-6,2014

12. M. Lebrun, A. Buades and J. M. Morel,“ A Nonlocal Bayesian Image Denoising Algorithm”, SIAM Journal on Imaging Sciences, vol. 6, no. 3, pp. $1665-1688,2013$

13. S. Mun and J. Fowler, "Block Compressed Sensing of Images Using Directional Transforms",In Proceedings of International 
Coference on Image Processing, Cairo, Egypt, January, pp.12-15,2013

14. X. Wu, X. Zhang and J. Wang, "Model-guided Adaptive Recovery of Compressive Sensing" , In Proceedings of Data Compression Conference, Snowbird, USA, April, 2009

15. J. Zhang, S. Liu, R. Xiong, "Improved Total Variation Based Image Compressive Sensing Recovery by Nonlocal Regularization", In Proceedings of IEEE International Symposium on Circuits and Systems, Beijing, China, July,pp.15-17,2013

16. J. Zhang, D. Zhao and W. Gao, "Group-based Sparse Representation for Image Restoration" ,IEEE Transactions on Image Processing, vol. 23, no. 8, pp. 3336-3351,2014

17. J. Zhang, D. Zhao, R. Xiong, S. Ma and W. Gao, "Image Restoration Using Joint Statistical Modeling in a Space-transform Domain”, IEEE Transactions on Image Processing, vol. 24, no. 6, pp. 915-928,2014

Cang Sheng received his B.S in Electronic and Information Engineering from Northeast Forestry University in 2004. He received his M.S. in Computer Application Technology from Northeast Forestry University in 2013. He is an Associate Professor in the School of Heilongjiang International University. His research interests include pattern recognition and intelligent control.

Achuan Wang got his Ph.D. in Machine Design Theory from Northeast Forestry University, Harbin, China in 2011. He is a professor in the School of Northeast Forestry University. His research interests include compressive sensing, intelligent control and pattern recognition. 